\title{
HIGH FREQUENCY OSCILLATIONS IN SS CYGNI
}

\author{
M.J. Arévalo ${ }^{1,2}$, J.E. Solheim ${ }^{3}$ and C. Lázaro ${ }^{1}$ \\ 1- Instituto de Astrofisica de Canarias, Spain \\ 2- Dept. Fisica, Universidad Politecnica de Canarias, Spain \\ 3- University of Tromsø, Norwe:
}

\section{INTRODUCTION}

Because of its relative brightness $\left(\mathrm{m}_{\mathrm{v}}\right.$ about 11.8 in quiescence and $\mathrm{m}_{\mathrm{v}}$ about 8.6 in outburst), SS Cygni is the Dwarf Novae most extensively studied since 1896 (Mattei et a1., 1985; Bath and van Paradijs, 1983).

Concerning its long-term behaviour, SS Cygni has short, long and anomalous outbursts. Correlations between outburst characteristics and periodicities have been studied by van Paradijs (1983), Campbel1 (1934), and Sterne and Campbe11 (1934). SS Cygni, as any cataclysmic variable, also presents rapid variations. Since the discovery of this variability (Warner and Robinson, 1972) many long runs of highspeed photometry have been performed for different Dwarf Novae, both during outbursts and in quiescence stages. Regarding to SS Cygni we can summarize these variations as:

Flickering: Irregular oscillations in brightness which are attributed to variations in the luminosity of the bright spot. The typical timescale is of a few minutes, and it is completely random and unpredictable (Robinson and Nather, 1979). The amplitude is about $0.1 \mathrm{mag}$, and the power spectrum shows a smooth decrease from low to high frequencies.

Quasi periodic oscillations: Periods of $31.5 \mathrm{~s}$ have been reported (Robinson and Nather, 1979) at maximum eruption. These decline in time periods of 150 $s$ and the mean amplitudes are $0.003 \mathrm{mag}$. It is assumed that they are associated to the accretion disk. Patterson (1981) found 32 and $36 \mathrm{~s}$ QPO's in outbursts.

Coherent oscillations: discovered by Patterson et a1. (1978) have a mean amplitude of $0.005 \mathrm{mag}$ and a determined period of about $10 \mathrm{~s}$ which can change rapidly

(Robinson and Nather, 1979). These oscillations are only present some times during outburst with variable amplitudes and period correlated with the magnitude. They have been related (Hildebrand et al., 1981) with the soft X-ray $9 \mathrm{~s}$ pulsations found by Cordoba et al. $(1980,1984)$, and may be due to damped oscillations on the surface of the white dwarf or to material orbiting close to the dwarf (Hildebrand et al., 1981).

It is also interesting to mention the periodicity of 12.18 minutes found by 
Bartolini et a1. (1985) and detected mainly in the red bands $R$ and $I$, that they take as support to the hypothesis that SS Cygni belongs to the intermediate polars group.

\section{OBSERVATIONS AND RESULTS}

We monitored SS Cygni during an extensive observing programme as part of an organized international campaign (Honey et al., 1988). The observations were carried out at the Observatorio del Roque de Los Muchachos (La Palma, Canary Islands), from September 9th to October 10th 1985. We used the $60 \mathrm{~cm}$ Swedish telescope and a one channel photometer modified by the Tromsø Astrophysics Group for continuous photometry, with a Johnson V-filter. The integration times were $5 \mathrm{~s}$ and $10 \mathrm{~s}$ in outbursts and quiescence respectively. Each run was occasionally interrupted for observations of the sky background and two nearby comparison stars. Two samples of the data are shown in Figure 1.

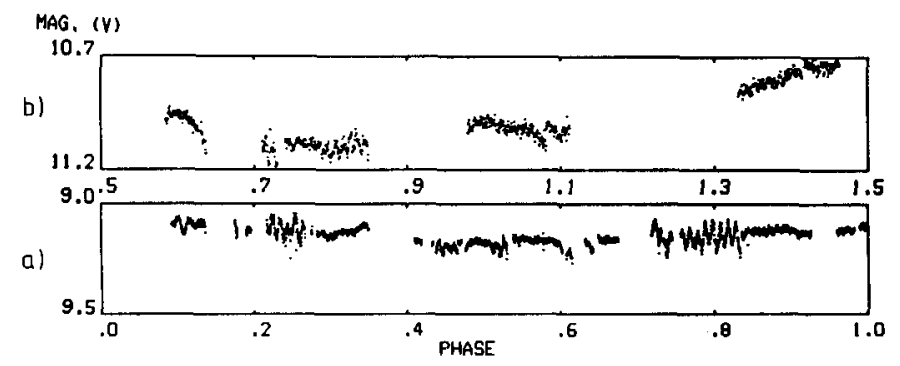

Figure 1: Two samples of the light curve observed. a) Sept. 10-11, (variations around phase .8 are due to telescope drive). b) Sept. 25-26.

The data reduction was carried out using a FT program at the University of Tromsø. The runs of both comparison stars were analyzed in order to exclude instrumental frequencies. During our observations SS Cygni had one eruption followed by a long quiescence period as shown in Figure 2.

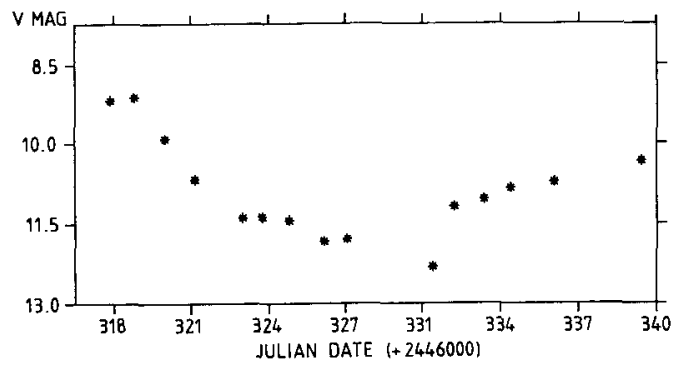

Figure 2: V-magnitude of SS Cygni during our observing period.

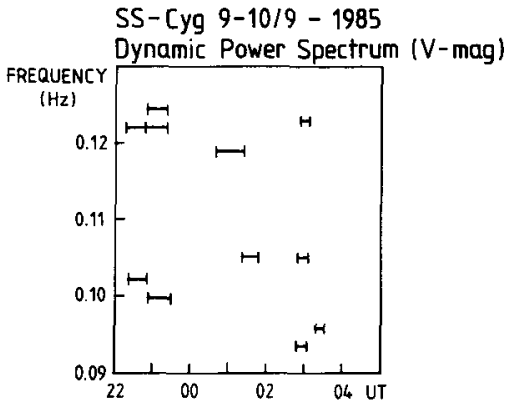

Figure 3: A dynamic power spectrum during the night sept. 9-10 1985. 
a) The highest frequency oscillations (8-10 s) were observed during the four nights at outburst, when the sampling rate was high enough. Figure 3 shows a dynamic power spectrum for one night. Two frequencies are present most of the time with some frequency drift towards slightly higher and lower frequencies. A consistent frequency increase with flux like that observed in the X-ray spectral range is not seen.

b) For the remaining of the observing nights we have searched for lower frequency oscillations in the range $0.4-60 \mathrm{~min}$. We find several periods with significant power even if they are not present for a11 the observing dates. Oscillations with periods in the range 30-33 s are present every night, with amplitudes reaching 0.003 mag, also well into the quiescent stage.

The complete results will be presented elsewhere.

\section{REFERENCES}

Bartolini, C., Guarnieri, A., Lolli, M., Piccioni, A., Giovannelli, F., Gaudenzi, S., and Lombardi, R.: 1985, "Multifrequency Behaviour of Galactic Accreting Sources", 50. Proceedings of the Frascati Workshop 1984, Ed. F. Giovannelli.

Bath, G.T. and Van Paradijs, J.: 1983, Nature 305, 33.

Campbe11, L.: 1934, Ann. Harvard Co11. Obs. 90, 93.

Cordoba, F.A., Chester, T.J., Tuohy, J.R., and Garmine, G.P.: 1980, Ap. J. 235, 163.

Cordoba, F.A., Chester, T.J., Mason, K.0., Kahn, S.M., and Garmire, G.P.: 1984, Ap. J. 278,739 .

Hildebrand, R.H., Spillar, E.J., and Stiening, R.F.: 1981, Ap. J. 243, 223.

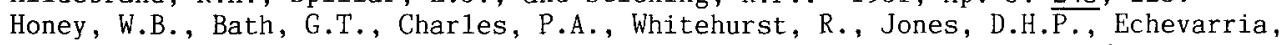
J., Arevalo, M.J., Solheim, J.E., Tovmassian, G., and Takagishi, K.: submitted to M.N.R.A.S.

Mattei, J.A., Saladya, M., Waagen, E.O., and Janes, C.M.: 1985, AAVSO Monograph No. 1.

Patterson, J.: 1981, Ap. J. Suppl. Ser. 45, 517.

Patterson, J., Robinson, E.L., and Kiplinger, A.L.: 1978, Ap. J. 226, L137.

Robinson, E.L. and Nather, R.E.: 1979, Ap. J. Supp1. Ser. 39, 461 .

Van Paradijs, S.J.: 1983, Astron. Ap. 125, L16.

Warner, B. and Robinson, E.L.: 1972, Nature Phys. Sci. 239, 2. 